\title{
The Interrelationships between Working Conditions, Job Satisfaction, Burnout and Mental Health among Hospital Physicians in Japan: a Path Analysis
}

\author{
Yasuharu TOKUDA ${ }^{1 *}$, Keiko HAYANO², Makiko OZAKI ${ }^{3}$, \\ Seiji BITO ${ }^{4}$, Haruo YANAI ${ }^{5}$ and Shunzo KOIZUMI6
}

\author{
${ }^{1}$ Center for Clinical Epidemiology, St. Luke's Life Science Institute, St. Luke's International Hospital, 9-1 \\ Akashi-cho, Chuo-ku, Tokyo 104-8560, Japan \\ ${ }^{2}$ Department of General Medicine, Kumamoto University School of Medicine, Kumamoto, Japan \\ ${ }^{3}$ Department of Epidemiology and Healthcare Research, Graduate School of Public Health and Medicine, \\ Kyoto University, Kyoto, Japan \\ ${ }^{4}$ Division of Clinical Epidemiology, National Hospital Organization Tokyo Medical Center, Tokyo, Japan \\ ${ }^{5}$ Department of Biostatistics, St. Luke's Graduate School of Nursing, Tokyo, Japan \\ ${ }^{6}$ Department of General Medicine, Saga University Faculty of Medicine, Saga, Japan
}

Received August 22, 2008 and accepted November 17, 2008

\begin{abstract}
A growing number of physicians are leaving their hospitals because of painful working conditions in hospitals throughout Japan. We set out to analyze the interrelationships between working conditions, job satisfaction, burnout and mental health among Japanese physicians. A cross-sectional survey was conducted in 2007 for hospital physicians throughout Japan. A path analysis based on structural equation modeling was utilized for examining the interrelationships between work control, on-call duty volume, job satisfaction (the Japan Hospital Physicians Satisfaction Scale), burnout (the Japanese version of the Maslach Burnout Inventory) and mental health (the General Health Questionnaire). Of 336 physicians who received a solicitation Email, 236 participated in our study (response rate, $70 \%$ ). Sixty physicians $(25.4 \%)$ were women with a mean age of $41 \mathrm{yr}$. In the path analysis, burnout and poor mental health were related directly to job dissatisfaction and short sleeping time, while they were related indirectly to poor work control and heavy on-call duty. In the multi-group path analysis of both genders, sleeping time was related to job satisfaction more likely among female physicians but less among male physicians. Healthcare policy makers need to implement immediate, extensive and decisive measures to improve work condition and to reduce overwork among hospital physicians.
\end{abstract}

Key words: Physician overwork, Work control, Job satisfaction, Burnout, Mental health

\section{Introduction}

Recent reports have shown that an increasing number of physicians throughout Japan are leaving their hospitals because of painful working conditions and restarting a new job with better working conditions in either private practice or part-time hospital practice ${ }^{1,2}$. These phe-

*To whom correspondence should be addressed. nomena are likely to become increasingly prevalent both in urban and rural hospitals in Japan. The mass media have reported that, because of a physician shortage, multiple urban hospitals are refusing to accept sick patients in ambulances and rural community hospitals have been shut down with a subsequent disruption of patient care.

Since burnout and poor mental health among hospital physicians are considered as major reasons for quitting a hospital work ${ }^{3)}$, it is important to elucidate the mecha- 
nisms causing work-related burnout and mental ill-health among hospital physicians at risk for resigning their current job ${ }^{1,2)}$ : furthermore, if burnout and poor mental health can be prevented, understanding the direct and indirect factors related to them are also important for implementing effective measures for preventing the collapse of the healthcare system in Japan. Given the importance of painful working conditions as likely main causes of burnout and poor mental health among hospital physicians, research addressing the working conditions, job satisfaction, burnout and mental health among Japanese hospital physicians is of extreme importance.

Williams et al. demonstrated that job dissatisfaction is directly related to burnout among physicians ${ }^{4)}$. Much research literature substantiates that poor working conditions, especially overwork and poor work control, are strongly related to dissatisfaction and burnout among physicians $^{3,5-8)}$ : moreover, physician dissatisfaction is also significantly associated with patient dissatisfaction ${ }^{9)}$ and low patient adherence to medical treatment ${ }^{10)}$.

In this study, we aimed therefore to analyze the interrelationships between working conditions, job satisfaction, burnout and mental health among Japanese physicians, using a path analysis technique which can delineate complicated causal pathways and quantitatively demonstrate whether an independent factor is directly or indirectly associated with an outcome with interest ${ }^{11}$. For measuring working conditions, we focused on poor work control and the number of on-call duty days or nights in this analysis, since these two factors are considered to be the main reasons cited by physicians leaving hospitals ${ }^{1,2}$.

\section{Subjects and Methods}

\section{Participants}

For this Japanese Physicians Work Life Survey, the study participants were recruited in September, 2007 by using multiple E-mail lists which are exclusively for Japanese hospital physicians working in hospitals (inpatient beds 220 ). The invitation E-mails, to participate in the survey, were sent to all physicians enrolled on these lists. The solicitation E-mail included a brief introduction describing the objectives of the study, as well as statements guaranteeing both the confidentiality and anonymity of responses. The Institutional Review Board of Kumamoto University School of Medicine approved the study.

\section{Instruments and data collection}

For evaluating job satisfaction, we used a section of the Japan Hospital Physicians Satisfaction Scale (JHPSS), which is a 28 -item instrument with the following six dimensions: relationship with other medical doctors; bur- den and business; community; relationship with co-medical staff; compensation; and, patient care issues. Responses to these items were rated using five-point Likert scales ranging from "strongly disagree" to "strongly agree". Reliability and validity of the JHPSS section we used was examined using a validation survey among Japanese physicians, with adequate psychometric properties; this validity and reliability have been previously demonstrated (Ozaki, Bito, Matsumura; Development of the Japan Hospital Physicians Satisfaction Scale, in press).

As for the instruments related to burnout in this study, we used the Japanese version of the Maslach Burnout Inventory ${ }^{12)}$. Its reliability and validity has been previously confirmed ${ }^{13)}$. The Maslach Burnout Inventory comprises 22 items with three subscales, including emotional exhaustion (EE), depersonalization (DP), and personal accomplishment (PA). The seven-point self-rated Likert scale responses ranged from "none" to "everyday" for the magnitude of frequency (0-6). Total scores of each subscale were calculated by adding up the scores of each item; however, since the PA subscale has been rarely used in terms of relating overwork and work stress to burnout, we utilized the EE and DP subscales in this study. For determining the prevalence of burnout, physicians with high EE scores ( $>27$ points) and high DP scores ( $>10$ points) were considered as in a burnout state.

For determining mental health, we used the 12-item version of the General Health Questionnaire (GHQ12), which was developed by Goldberg to evaluate changes in psychological condition ${ }^{14)}$. Possible answers to each question were as follows: not at all/better than usual; same as usual; worse than usual; and, much worse than usual. Total scores were calculated by adding up each score. A higher total score indicated the poorer mental health.

Work control was evaluated using a self-rated magnitude of controllability question regarding day-to-day aspects of practice in multiple settings, based on study items prepared by and analyzed by McMurray et $a l^{15}$. We asked: "How much control do you have over each of the following?" The work-setting items included the following: selecting physicians for referrals; deciding when to admit to the hospital; determining length of hospital stay; prescription contents; details of office scheduling; selecting diagnostic tests; paperwork volume; work hours; unexpected work volume; volume of patient load (inpatients plus outpatients); work issues (office space and facilities); and, control of working pace. The four-point self-rated Likert scale responses ranged from "slight or none" to "extreme".

Regarding the reliability of the instruments, Cronbach's Alpha Coefficient was 0.83 for job satisfaction items, 0.89 for emotional exhaustion items and 0.77 for emotional exhaustion items relating to burnout, 0.87 for mental sta- 
tus items, and 0.83 for work control measurement items. The questionnaires also included items related to demographics (age and gender), specialty, the number of oncall duty (average days of overnight work plus weekend work in a month), and the average daily sleeping time by considering both weekdays and weekends (hours/day).

\section{Statistical analysis}

Based on our hypothesis that the mechanism causing burnout and poor mental health among Japanese physicians was due to poor work control, overwork, sleep deprivation and poor job satisfaction, a path model was developed utilizing a structural equation modeling procedure with maximum likelihood estimations for path coefficients ${ }^{16}$. Given the degree of association between independent and dependent variables in our dataset, this procedure produced the best fit model including only significant path coefficients ${ }^{11)}$. We constructed the model with a sequential pathway from EE to DP and another pathway from EE to poor mental health, based on published results indicating that the probable progression was from an EE state to a DP state ${ }^{17)}$.

Unstandardized path coefficients can be used for estimating their effects between the real variables. For instance, if the unstandardized path coefficient was one, we can estimate that the increase in one unit of causative variable is related to the increase in 1 unit of outcome variable. On the other hand, standardized path coefficients can be used for comparing them by the strength of association between different paths represented in terms of standardized variables.

Multi-group path analysis of the two groups, men and women, was conducted and was compared to the first model of all physicians, using several fit measures. In this multi-group analysis, path coefficients were also estimated separately for men and women. The fit measures included relative $\chi^{2}$, comparative fit index (CFI), and root mean square error of approximation (RMSEA), as these are the most commonly used indices ${ }^{16)}$. Detailed description of fit indices in structural equation modeling can be found in the textbook chapter by Yuan and Bentler ${ }^{16)}$. The relative $\chi^{2}$ is calculated by model $\chi^{2} /$ degree of freedom (df) and this statistic increases with a larger sample size and thereby a higher chance of the significance. The CFI evaluates the relative fitness by comparing the substantive model to a base model about the better explanation for the covariance structure. CFI values $>0.90$ indicate good fit of the model ${ }^{16)}$. The root mean square error of approximation (RMSEA) estimates the lack of fit in a model compared to a saturated model. Values of RMSEA $<0.10$ is indicative of a good model ${ }^{16)}$. STATA version 10 (Stata Corp, College Station, TX, USA) was used for descriptive statistics and AMOS version 7.0 (SPSS-Japan,
Tokyo, Japan) was used for structural equation modeling. A two-tailed $p$-value $<0.05$ was considered statistically significant.

\section{Results}

We distributed questionnaires by mail to 336 physicians who had responded positively to the solicitation E-mail. We received the written responses from 236 physicians (response rate, $70.2 \%)$. Of all respondents: 60 (25.4\%) were women; 75 (31.8\%) were generalist physicians (primary care or general internal medicine); the mean age was $40.9 \mathrm{yr}$ (range, 26 to 67 ; SD, 7.8); and postgraduate clinical experience was between 3 to $42 \mathrm{yr}$ (mean, 15.8; SD, 7.4).

The mean number of on-call duty was 6.0 in a month (range, 0 to 20) and the mean of average sleeping time was $6 \mathrm{~h}$ in a day (range, 3 to 8; SD 0.9). The majority $(89.0 \%)$ of the participants claimed that their annual salary was 5 million Japanese yen or less (1 US Dollar $=118$ Japanese yen in average rate of 2007). Mean score for emotional exhaust was 23.4 (SD, 11.6) and that for depersonalization was 7.0 (SD, 5.9). Correlation coefficient was 0.65 between emotional exhaust and depersonalization scores. Among the total of 336 physicians, 19\% were considered to be in a burnout state.

By analyzing the paths between work control, job satisfaction, EE and DP of burnout, and mental health along with on-call duty and daily sleeping time, path analyses produced a model with significant path coefficients (Fig. 1). The standardized coefficients shown in Fig. 1 are all statistically significant. Table 1 shows maximumlikelihood estimates of path coefficients (unstandardized). These results suggest that there are the pathways from work control and on-call duty through sleeping time and

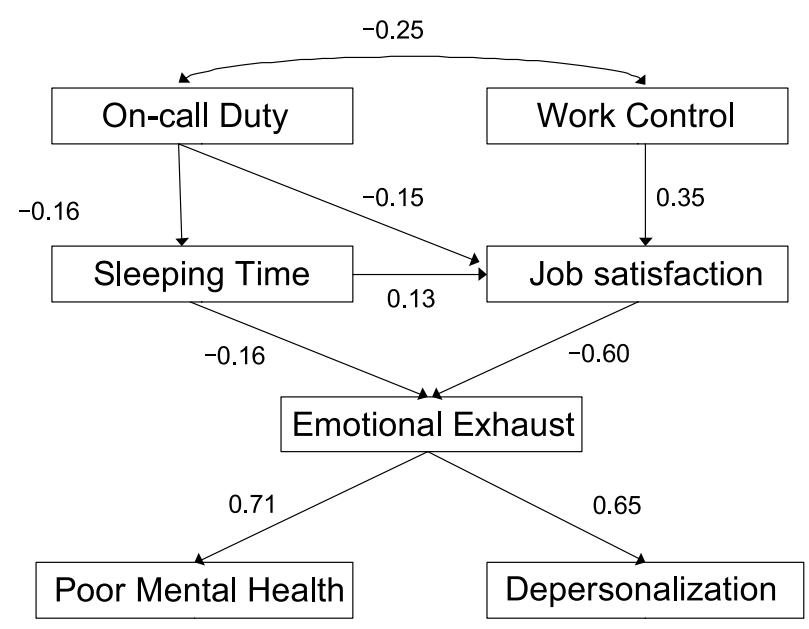

Fig. 1. Path model with the standardized coefficients in all physicians. 
Table 1. Maximum-likelihood estimates of path coefficients for path analysis among all physicians

\begin{tabular}{lllccr}
\hline & Path & & Coefficient & (unstandardized) & $p$-value \\
& & & estimate & SE & \\
\hline On-call duty & to & Sleeping time & -0.410 & 0.016 & 0.012 \\
Work control & to & Job satisfaction & 0.418 & 0.072 & $<0.001$ \\
Sleeping time & to & Job satisfaction & 0.990 & 0.458 & 0.031 \\
On-call duty & to & Job satisfaction & -0.296 & 0.121 & 0.015 \\
Job satisfaction & to & Emotional exhaust & -0.106 & 0.009 & $<0.001$ \\
Sleeping time & to & Emotional exhaust & -0.219 & 0.070 & 0.002 \\
Emotional exhaust & to & Depersonalization & 0.602 & 0.046 & $<0.001$ \\
Emotional exhaust & to & Poor mental health & 0.250 & 0.016 & $<0.001$ \\
\hline
\end{tabular}

$\mathrm{SE}=$ standard error

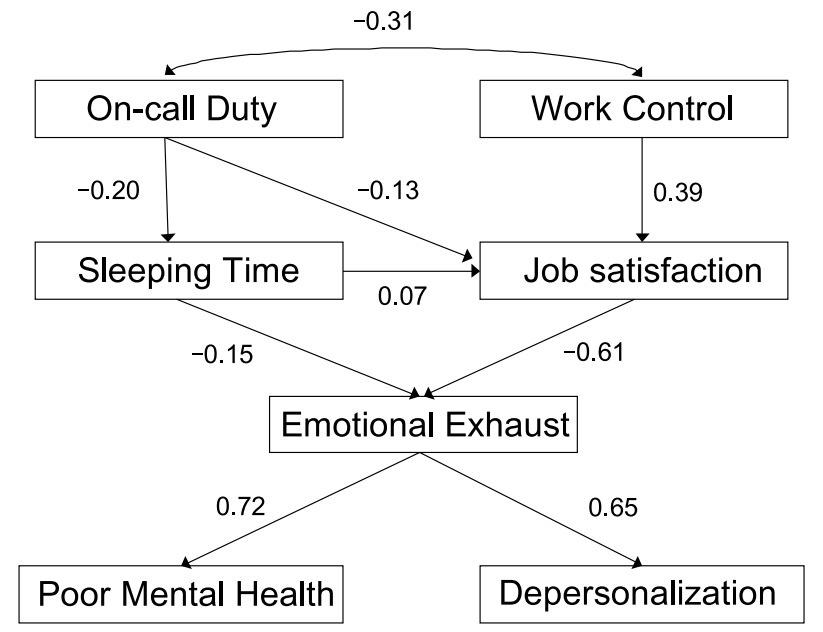

Fig. 2. Path model with the standardized coefficients in male physicians.

job satisfaction to burnout and poor mental health. Thus, burnout and poor mental health were directly related to job dissatisfaction and short sleeping time and burnout and poor mental health were indirectly related to poor work control and heavier on-call duty in Japanese physicians.

In addition, based on the relative magnitudes of the standardized coefficients, work control and job satisfaction were stronger factors for burnout than on-call duty or sleeping time.

Next, the multi-group path analysis was constructed to generate separate subgroup models of men (Fig. 2) or women (Fig. 3). Path coefficients obtained by the multigroup path analysis are shown in Table 2 . The path coefficients from sleeping time to job satisfaction were still statistically significant among women but not so among men. Thus, sleeping time was related to job satisfaction more likely among female physicians but less so among male physicians. Table 3 shows goodness-of-fit statistics between the model of all physicians combined and that of multi-group path analyses. $\chi^{2} / \mathrm{df}$ and comparative fit

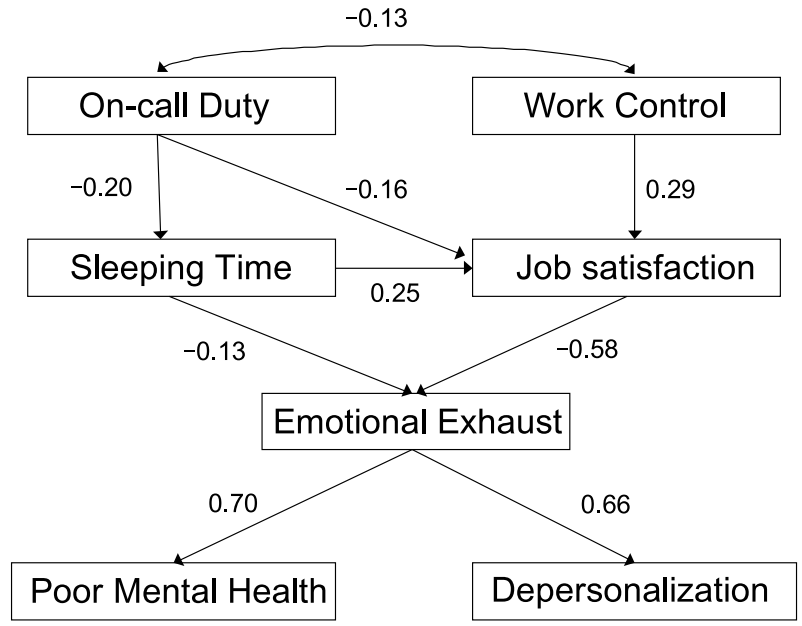

Fig. 3. Path model with the standardized coefficients in female physicians.

index of the model of all physicians were all greater (better fit) than those obtained from the multi-group path analysis model. The root mean square error of approximation was smaller (better fit) in the multi-group path analysis model than in the model of all physicians combined. Thus, it may be better to use the model of all physicians combined as a reasonable representative mechanism for covering both genders of Japanese physicians.

\section{Discussion}

Our path model indicates that poor work control and heavy on-call duty are directly associated with job dissatisfaction and short sleeping time and indirectly associated with burnout and poor mental health. This is the first path analysis study among Japanese physicians to show the pathways to burnout and mental ill-health from poor working conditions as fundamental root causes. Poor work control can lead to job dissatisfaction, while heavy on-call duty can cause job dissatisfaction and short sleeping time; furthermore, job dissatisfaction and short 
Table 2. Maximum-likelihood estimates of path coefficients for multi-group path analysis

\begin{tabular}{|c|c|c|c|c|c|}
\hline \multirow[t]{2}{*}{ Men } & \multirow[b]{2}{*}{ Path } & & \multicolumn{2}{|c|}{ Coefficient (unstandardized) } & \multirow[t]{2}{*}{$p$-value } \\
\hline & & & estimate & SE & \\
\hline On-call duty & to & Sleeping time & -0.050 & 0.019 & 0.008 \\
\hline Work control & to & Job satisfaction & 0.472 & 0.085 & $<0.001$ \\
\hline Sleeping time & to & Job satisfaction & 0.583 & 0.568 & 0.304 \\
\hline On-call duty & to & Job satisfaction & -0.264 & 0.149 & 0.077 \\
\hline Job satisfaction & to & Emotional exhaust & -0.105 & 0.010 & $<0.001$ \\
\hline Sleeping time & to & Emotional exhaust & -0.215 & 0.082 & 0.009 \\
\hline Emotional exhaust & to & Depersonalization & 0.597 & 0.053 & $<0.001$ \\
\hline Emotional exhaust & to & Poor mental health & 0.264 & 0.019 & $<0.001$ \\
\hline \multirow[t]{2}{*}{ Women } & & & \multicolumn{2}{|c|}{ Coefficient (unstandardized) } & $p$-value \\
\hline & Path & & estimate & SE & \\
\hline On-call duty & to & Sleeping time & -0.055 & 0.035 & 0.121 \\
\hline Work control & to & Job satisfaction & 0.340 & 0.137 & 0.013 \\
\hline Sleeping time & to & Job satisfaction & 1.711 & 0.805 & 0.034 \\
\hline On-call duty & to & Job satisfaction & -0.305 & 0.226 & 0.177 \\
\hline Job satisfaction & to & Emotional exhaust & -0.114 & 0.021 & $<0.001$ \\
\hline Sleeping time & to & Emotional exhaust & -0.171 & 0.140 & 0.222 \\
\hline Emotional exhaust & to & Depersonalization & 0.629 & 0.093 & $<0.001$ \\
\hline Emotional exhaust & to & Poor mental health & 0.216 & 0.029 & $<0.001$ \\
\hline
\end{tabular}

$\mathrm{SE}=$ standard error.

Table 3. Goodness-of-fit statistics for the models of all physicians and of multi-groups (men and women)

\begin{tabular}{lcc}
\hline Statistic & Analysis of all physicians & Multi-group analysis \\
\hline Degree of freedom (df) & 12 & 24 \\
$\chi^{2}$ & 66.0 & 83.7 \\
$\chi^{2} / \mathrm{df}$ & 5.50 & 3.49 \\
Comparative fit index & 0.902 & 0.893 \\
Root mean square error of approximation & 0.138 & 0.103 \\
\hline
\end{tabular}

sleeping time can result in burnout and poor mental health ${ }^{18)}$. Work control and job satisfaction seem stronger factors for burnout than on-call and sleeping time. Thus, it is important to implement concrete and decisive measures for improving work control and preventing overwork among Japanese physicians.

A review of existing literature indicates that there is a strong positive association existing regarding physician dissatisfaction, burnout, and physician turnover. Hospitals with higher levels of physician dissatisfaction have resulting higher physician turnover rates ${ }^{5)}$. Some adverse effects of higher physician turnover rates include discontinuity of the care provided by physicians to patients, instability of the patient-physician relationship, institutional unpredictability, an actual shortage of physicians, and eventually departmental and hospital shutdowns ${ }^{19)}$.

Indeed, multiple observations, including governmental reports and mass media reports, remark that the Japanese healthcare system is currently at risk of collapse because of the rapid increase in physician resignations in hospitals throughout Japan ${ }^{1)}$. In addition, an increasing number of hospital physicians have committed suicide recently and Japanese courts have ruled that the reasons of the suicides, in many cases, were due to their overwork ("Karoshi" in Japanese) and the courts ordered hospital administrators to compensate the bereaved families ${ }^{2,20)}$. Improving working conditions is critical for preventing further massive resignations of physicians and for maintaining a durable healthcare system in Japan. In particular, politicians and policy makers should be informed on these crucial findings and causal relationships, since immediate and extensive measures can be implemented more effectively by those responsible for regulating and controlling physicians' work at the national level.

The multi-group analysis of both genders suggested that short sleeping time is more important for job dissatisfaction among female physicians than among male physi- 
cians. A possible lower tolerance to sleep deprivation among women than among men may explain the difference of effects of sleeping time on job satisfaction ${ }^{21)}$. Another possibility was that men had less willingness to admit to fatigue than women.

Thus, ensuring enough sleeping time is important for improving job satisfaction for female physicians particularly among specialties, such as pediatrics and obstetrics/gynecology, in which many female physicians are working; however, the Japanese government estimates that there are only 2.5 pediatricians per pediatric department at hospitals with pediatric departments, and that $97 \%$ of these pediatricians work consecutively for $32 \mathrm{~h}$ or more when they are on call not only in- hospital call but also at-home call ${ }^{22}$. In addition to the $32 \mathrm{~h}$ or more workdays, they are on call every second or third days on average because of the vicious cycle resulting from the shortage of physicians in this area ${ }^{22)}$. Regarding short sleeping time, there may be a possibility that some physicians have insomnia rather than sleep deprivation. Heavy oncall duty and/or poor work control could lead to depression with insomnia or poor-quality sleep among physicians $^{23,24)}$. Depressed physicians may report that they had short sleeping time but this shortness of sleeping time would derive from insomnia but not from sleep deprivation. Thus, we may also need to consider possible mood disturbances in physicians with short sleeping time.

There are several limitations in our study. Firstly, the study design is cross-sectional and the sample size is relatively small. Thus, our findings may have inferential and generalizability limits. Prospective longitudinal and a larger-sample study may be needed for confirming the effects of work control and overwork on multiple dimensions of physician psychological well-being; however, since a growing number of reports, including this one, indicate that physician overwork and poor work control in Japan are evident and urgent, swift and extensive measures should be implemented for preventing what could become a healthcare collapse.

Secondly, there may be sampling bias, due to the recruitment of the sample by E-mail solicitation from participants in multiple E-mail lists. Data on the exact number of subscribers to each e-mail list could not be obtained. Many of the physicians choosing to participate in the survey might have done so because of their poor working conditions and consequential high interest in work issues or because of their burnout state and accompanying higher interest in mental health issues.

Thirdly, although we used the number of on-call duty days or nights as a general indicator of physician overworking, additional factors are also likely to contribute to overwork among Japanese physicians. These factors include the unpaid extra overtime work after the end of the working day, the midnight telephone calls from hospital staff because of the lack of an in-charge system for communicating the unexpected events related to patient care, the obligation to keep a cellular phone on $24 \mathrm{~h}$ a day, $7 \mathrm{~d}$ a week and the obligation to remain within a set geographic area $24 \mathrm{~h}$ a day, $7 \mathrm{~d}$ a week. These existing working conditions have already caused deleterious effects on the job satisfaction and mental health of Japanese physicians; therefore, including these multifaceted working condition measurements into an analysis is needed for further clarifying the true risk factors for job dissatisfaction and poor mental health among physicians.

In conclusion, despite several limitations, our results demonstrate causal pathways from poor work control and heavy on-call duty through job dissatisfaction and short sleeping time to burnout and poor mental health. Short sleeping time is an especially important factor for job dissatisfaction among women more so than among men. Work control and job satisfaction are more likely to be important for burnout than on-call and sleeping time. Many Japanese hospitals are likely to have high levels of physician dissatisfaction, burnout, or poor mental health and thus have higher physician turnover rates and therefore be at a higher risk of having a hospital shutdown occur. For preventing a massive collapse in the healthcare system throughout Japan, politicians and policy makers should implement immediate, extensive and decisive measures to improve work control and reduce overwork among physicians working in Japanese hospitals.

\section{References}

1) Komatsu H (2006) Iryouhoukai. Asahi Shimbun, Tokyo (in Japanese).

2) Tanabe I (2007) Document Iryoukiki. Asahi Shimbun, Tokyo (in Japanese).

3) Williams ES, Skinner AC (2003) Outcomes of physician job satisfaction: a narrative review, implications, and directions for future research. Health Care Manage Rev 28, 119-39.

4) Williams B (2002) Physician stress \& burnout. Tenn Med 95, 445-51.

5) Freeborn DK (2001) Satisfaction, commitment, and psychological well-being among HMO physicians. West J Med 174, 13-8.

6) Maslach C, Schaufeli WB, Leiter MP (2001) Job burnout. Annu Rev Psychol 52, 397-422.

7) Edwards N, Kornacki MJ, Silversin J (2002) Unhappy doctors: what are the causes and what can be done? BMJ 324, 835-8.

8) Wetterneck TB, Linzer M, McMurray JE, Douglas J, Schwartz MD, Bigby J, Gerrity MS, Pathman DE, Karlson D, Rhodes E (2002) Worklife and satisfaction of general internists. Arch Intern Med 162, 649-56.

9) Haas JS, Cook EF, Puopolo AL, Burstin HR, Cleary 
PD, Brennan TA (2000) Is the professional satisfaction of general internists associated with patient satisfaction? J Gen Intern Med 15, 122-8.

10) DiMatteo MR, Sherbourne CD, Hays RD, Ordway L, Kravitz RL, McGlynn EA, Kaplan S, Rogers WH (1993) Physicians' characteristics influence patients' adherence to medical treatment: results from the Medical Outcomes Study. Health Psychol 12, 93-102.

11) Gefen D, Straub D, Boudreau M (2000) Structural Equation Modeling and Regression: guidelines for Research Practice. Structural Equation Modeling 4, 7.

12) Maslach C, Jackson SE (1981) The measurement of experienced burnout. J Occup Beh 2, 99-113.

13) Higashiguchi K, Morikawa $Y$, Miura K, Nishijo $M$, Tabata M, Yoshita K, Sagara T, Nakagawa H (1998) The development of the Japanese version of the Maslach Burnout Inventory and the examination of the factor structure. Nippon Eiseigaku Zasshi 53, 447-55.

14) Goldberg DP (1972) The detection of psychiatric illness by questionnaire; a technique for the identification and assessment of non-psychotic psychiatric illness. Oxford University Press, London.

15) McMurray JE, Linzer M, Konrad TR, Douglas J, Shugerman R, Nelson K (2000) The work lives of women physicians results from the physician work life study. The SGIM Career Satisfaction Study Group. J Gen Intern Med 15, 372-80.

16) Yuan K, Bentler P (2007) Structural Equation Modeling. In: Handbooks of Statistics Vol.26: Psychometrics, Rao CR, Sinharay S (Eds.), 297-358, Elsevier, Amsterdam.
17) Stout JK, Williams JM (1983) Comparison of two measures of burnout. Psychol Rep 53, 283-9.

18) Killgore WD, Kahn-Greene ET, Lipizzi EL, Newman RA, Kamimori GH, Balkin TJ (2008) Sleep deprivation reduces perceived emotional intelligence and constructive thinking skills. Sleep Med 9, 517-26.

19) Lichtenstein RL (1984) The job satisfaction and retention of physicians in organized settings: a literature review. Med Care Rev 41, 139-79.

20) Fujino $Y$, Horie S, Hoshuyama $T$, Tsutsui $T$, Tanaka $Y$ (2006) A systematic review of working hours and mental health burden. Sangyo Eiseigaku Zasshi 48, 87-97 (in Japanese).

21) Gay CL, Lee KA, Lee SY (2004) Sleep patterns and fatigue in new mothers and fathers. Biol Res Nurs 5, 311-8.

22) Ehara A (2005) Only 2.5 pediatricians per pediatric department of Japanese hospital: can each hospital continue to provide a pediatric emergency service? BMJ Rapid Responses (28 September) http://www.bmj.com/ cgi/eletters/331/7518/648\#117657. Accessed February 11, 2008.

23) Rosen IM, Gimotty PA, Shea JA, Bellini LM (2006) Evolution of sleep quantity, sleep deprivation, mood disturbances, empathy, and burnout among interns. Acad Med 81, 82-5.

24) Handel DA, Raja A, Lindsell CJ (2006) The use of sleep aids among Emergency Medicine residents: a web based survey. BMC Health Serv Res 6, 136. 